\title{
Sobre la determinación y resolución de ambigüedades: entre la lingüística y la mecánica cuántica.
}

On the determination and resolution of ambiguities: between linguistics and quantum mechanics.

Nalliely Hernández Cornejo

Departamento de Filosofía. Universidad de Guadalajara (MÉXICO)

CE: nallie3112@hotmail.com

Raúl E. Rodríguez Monsiváis

Departamento de Filosofía. Universidad de Guadalajara (MÉXICO)

CE: siembra@hotmail.com

DOI: $10.32870 /$ sincronia.axxiv.n77.4a20

Esta obra está bajo una Licencia Creative Commons Atribución-NoComercial 4.0 Internacional

$B Y \cdot N C$

Recibido: 05/06/2019

Revisado: $27 / 09 / 2019$

Aprobado: $12 / 11 / 2019$

\section{RESUMEN}

El objetivo de este trabajo es defender la siguiente tesis: existe un paralelismo en el tratamiento de las ambigüedades en lingüística y en del uso de los conceptos de onda y partícula en la mecánica cuántica, este paralelismo consiste en que en última instancia hay un contexto que juega un papel central en la desambiguación. Esta tesis la justificamos atendiendo a la definición de "paralelismo" y en virtud de dos premisas: la primera según la cual pese a que la ambigüedad puede deberse y explicarse en función de algunos de los diferentes niveles de organización de la lengua, es en el contexto de emisión de una expresión que se determina una interpretación dada. La segunda 
premisa enuncia que mientras el electrón pueda concebirse como una onda o como una partícula, es en el contexto de experimentación que se elegirá una interpretación dada. Además, intentaremos explicar esta última ambigüedad en función de la transición en la teoría física entre el lenguaje formal y el lenguaje natural u ordinario y los distintos principios de realidad que ambos suponen, así como dar una interpretación no dualista entre naturaleza y lenguaje a partir de ella.

Palabras clave: Ambigüedad. Niveles de organización de las lenguas. Contexto lingüístico. Contexto experimental. Dualidad onda-partícula.

\section{ABSTRACT}

The aim of this work is to defend the following thesis: there is a parallel in the treatment of ambiguities in linguistics and the use of wave and particle concepts in quantum mechanics, this parallelism is that ultimately there is a context that it plays a central role in disambiguation. This thesis is justified according to the definition of "parallelism" and by virtue of two premises: the first one according to which although ambiguity can be due and explained according to some of the different levels of organization of the language, it is in the emission context of an expression that a given interpretation is selected. The second premise states that while the electron can be conceived as a wave or as a particle, it is in the context of experimentation that a given interpretation will be chosen. In addition, we will try to explain this last ambiguity in terms of the transition in physical theory between formal language and natural or ordinary language and the different principles of reality that both imply, as well as giving a non-dualistic interpretation between nature and language from she.

Keywords: Ambiguity, linguistic context, experimental context, wave-particle duality, linguistic organizational levels.

\section{Introducción.}

El presente trabajo tiene el objetivo de mostrar que existe un paralelismo entre el estudio de las lenguas naturales respecto a las ambigüedades y en mecánica cuántica en lo concerniente a la 
interpretación onda-partícula en el estudio del electrón, donde en ambos sectores el contexto juega un papel primordial en la desambiguación. Para lograr dicho objetivo procederemos de la siguiente manera. Primero, entendemos por "paralelismo" simplemente alguna correspondencia, semejanza o equivalencia entre dos cosas o fenómenos. Así, por ejemplo podemos mantener que hay un paralelismo entre una casa y un carro debido que ambas poseen una equivalencia estructural, ambas tiene puertas, ventanas, entre otras, también son funcionalmente semejantes ya que son elaboraciones del hombre para uso humano. Ahora bien, en el segundo apartado se presentará evidencia exhaustiva para legitimar nuestra primer premisa, según la cual la ambigüedad lingüística se puede explicar apelando a los diferentes niveles de organización lingüística, a saber, el nivel fonológico, el nivel morfosintáctico, el nivel léxico, la dimensión pragmática, así como a aspectos de la lógica. Sin embargo, pese a que la ambigüedad se explique en función de esos niveles, hay razones para sostener que el contexto de emisión de una construcción es donde se selecciona una interpretación. En segundo lugar, para justificar nuestra segunda premisa, según la cual el contexto de experimentación determina la interpretación corpuscular u ondulatoria del electrón, se reconstruye brevemente el proceso conceptual que llevó a la interpretación dual de los objetos atómicos, como onda o como corpúsculo, establecida en el principio de complementariedad. Asimismo, profundizaremos en cómo esta dualidad expresa una ambigüedad en el uso de los conceptos, por tanto, en la identidad de los objetos, que se resuelve gracias al contexto experimental y que, a su vez, implica que la descripción ondulatoria o corpuscular del electrón o el fotón son ambas necesarias, pero mutuamente excluyentes. Daremos así cuenta de la semejanza entre el uso de los conceptos en física atómica que se efectúa desde esta perspectiva y la ambigüedad lingüística en las lenguas naturales.

Por último, nos detendremos brevemente en delimitar y explicar el paralelismo en ambos campos e intentaremos plantear algunos problemas y posibles vías de solución epistémicos y lingüísticos que se pueden derivar de éste en torno a la naturaleza de las ambigüedades en las descripciones, la relación entre lenguaje y naturaleza o el concepto de objetividad. 


\section{La ambigüedad en las lenguas naturales.}

La ambigüedad es un fenómeno que ocurre en las lenguas naturales, consiste en el hecho de que una sola construcción lingüística expresa más de un sentido o contenido semántico, esto es, cuando posee más de un significado. Técnicamente una construcción lingüística o emisión es ambigua si puede ser interpretada de más de una manera (Löbner 2002, p. 41). Debido a que se trata de la variación del significado de las construcciones lingüísticas la ambigüedad es un fenómeno fundamentalmente semántico, aunque esta ambigüedad esté determinada por diferentes aspectos de los niveles de organización lingüística. Así, hay ambigüedades fónicas, morfosintácticas, léxicas, pragmáticas y lógicas. Es decir, que el procedimiento que sirve para desambiguar consiste en apelar a alguno de estos niveles de organización de las lenguas. Otro rasgo esencial de la ambigüedad consiste en que se trata de un fenómeno en que dos o más interpretaciones se presentan de manera disyuntiva, por lo que los diversos contenidos semánticos relativos a una sola construcción lingüística se excluyen entre sí.

\subsection{Ambigüedad fónica}

En gran medida la mayoría son morfonológicas y por homofonía. Se trata de una construcción que al ser emitida por el sonido que se produce puede ser interpretada de diferentes maneras. Pongamos por ejemplo los siguientes casos:

(1)

a. Me dirán las sílabas del verso.

b. Medirán las sílabas del verso.

(2)

a. Tu juego de mente

b. Tu juego demente. 
Un oyente que esté ante una emisión de cualquiera de las construcciones que dan como resultado (1) o (2) puede encontrarse ante la disyuntiva de cuál de las dos construcciones a. o b. se está comunicando. En estos casos el contexto juega un papel determinante en la selección de lo que se está profiriendo. En (1) ya sea si se está solicitando que le digan las sílabas que hay en un verso o bien que cuenten la cantidad de sílabas que hay en un verso. En (2) si se trata de un juego que involucra capacidades cognitivas o intelectuales, o bien si se trata de un juego demencial o de locos, por ejemplo la famosa ruleta rusa en el que se dispone de una bala a un revólver y se hace rodar al azar para posteriormente dispararla apuntando a la propia cabeza.

\subsection{Ambigüedad sintáctica}

Este tipo de ambigüedades se deben a las relaciones de dependencia o de determinación que se dan entre los componentes de una construcción lingüística. Atendamos los siguientes casos:

(3) El profesor de inglés argentino.

En este caso el adjetivo "argentino" puede estar modificando al sintagma [el profesor de inglés] de tal modo que su significado sería que el profesor de inglés es de nacionalidad argentina. Pero, ese mismo adjetivo "argentino" puede ser parte de la frase preposicional [de inglés argentino] que en tal caso estaría modificando al sintagma nominal determinado [el profesor], siendo así las cosas "argentino" modifica a "inglés" de modo que se estaría hablando de un tipo de inglés o la forma en que los argentinos pronuncian característicamente el inglés que se distingue del "inglés coreano", "el inglés mexicano" y "el inglés francés".

(4) Mario vio al hombre con los binoculares.

En este caso el sintagma preposicional [con los binoculares] puede ser determinante del sustantivo "hombre" de modo que se puede interpretar con la paráfrasis que Mario vio al hombre que tenía o 
portaba binoculares. Pero, el mismo sintagma preposicional puede ser un circunstancial de instrumento del verbo "ver", en tal caso se interpretaría como que Mario usó los vinculares para ver al hombre.

(5) Juan y María están casados.

Esta construcción es ambigua debido a que no es claro si el predicado es sobre dos sustantivos coordinados por separado [[Juan] y [María] están casados] o si es un sintagma coordinativo [[Juan y María] están casados]. Esta segunda interpretación sería que están casados entre ellos, en tanto que la primera sería que están casados pero cada cual con su pareja, no entre ellos.

\subsection{Ambigüedad léxica}

Tal vez este tipo de ambigüedad sea la que más se produce en las lenguas debido a que una misma palabra por lo general posee más de un significado, lo que técnicamente se denomina polisemia o un mismo significado es capturado por diversas palabras, lo que se conoce como sinonimia, y también una misma forma léxica puede tener diferentes significados, lo que ha recibido el nombre de homonimia. Este es un tipo de relaciones semánticas que eventualmente implican o conducen a ambigüedades. Las relaciones léxico- semánticas en las lenguas son de muchos a muchos, por ello es donde se encuentra la mayor variabilidad y diversidad.

Así es el comportamiento de las lenguas que incluso se dan relaciones entre la polisemia y la sinonimia. La polisemia se da en un término y en la relación con sus diferentes significados o acepciones. La sinonimia es una relación entre términos diferentes que comparten un significado, ya sea manera parcial o total. Por ello, es de esperarse que dos términos léxicos puedan coincidir en algunas de sus acepciones, lo que es relativo a su polisemia, conformando así una relación de sinonimia entre ellas. Por ejemplo, y a diferencia de lo que mantiene Alonso Ramos (2011, pp. 340346), de donde son tomados los ejemplos, hay polisemia en el verbo "destapar" cuyo uno de sus significados es "quitar la tapa de algo que la tiene, de modo que se quita la cubierta dejando el 
contenido descubierto. Así, se dice "destapó la botella". Pero de este significado se deriva otro más abstracto que tiene que ver con el hecho de poner al descubierto un hecho oculto que se hace público. Por ello se dicen cosas como "el periodista destapó los casos de corrupción". Por su parte, "descubrir" tiene como uno de sus significados el hecho de hacer aparecer un objeto que está cubierto por algo. Tomando en cuenta este significado se expresan cosas como "el presidente descubrió la estatua". A su vez, tiene el significado relacionado con el anterior de hacer público un hecho que estaba encubierto. Esto hace que se digan cosas como "el presidente descubrió el complot". La polisemia tanto de "destapar" como de "descubrir" las hace coincidir en esa segunda acepción, lo que las hace sinónimas junto con construcciones como "sacar a la luz", "dar a conocer", "revelar", entre otras. Esto hace que a veces pueden sustituirse sin alterar el contenido semántico de la construcción en la que aparecen "Mario destapó/descubrió casos de corrupción”, pero otra veces no "Mario destapó/*descubrió la olla para probar la comida". Pero, así como se dan estas relaciones se dan otras que implican o determinan ambigüedades. Veamos los siguientes ejemplos.

(6) Pedro le compró un carro a su suegra.

En este ejemplo, las interpretaciones son, por un lado que el beneficiario de la compra fue la suegra $y$, por otro lado que la suegra fue la fuente de la compra. Estas interpretaciones se deben a la composición que forman el verbo "comprar" y la preposición "a". El verbo "comprar" semánticamente se compone de un comprador (o vendedor) y alguien que compra, bajo esta lectura la preposición "a" indica el vendedor o comprador, donde el rol semántico del SP [a su suegra] es el de fuente de la compra. Con la proposición "a" y el verbo "comprar" se señala una transferencia, la del carro que va de la suegra a Pedro mediante la compra. Esto no funcionaría si la composición fuera entre "regalar" y "a", que es la otra lectura, donde "a" es direccional e indica que la transferencia del carro va de Pedro a su suegra, donde "su suegra" tiene un rol semántico de beneficiario y esto debido a que un objeto comprado puede a su vez ser transferido o dado a otra persona. 
(7) Mi novio entró al bar y luego luego comenzó a coquetear con su amigo.

Aquí la ambigüedad la introduce la preposición "con" que bajo una de sus acepciones es equivalente a la preposición "a" con significado direccional. En este sentido el coqueteo del novio va dirigido a su amigo, lo que produce una lectura graciosa pues supone una relación homosexual. Sin embargo, otro de los significados de la preposición "con" es el de compañía, bajo esta lectura se da a entender que el coqueteo de su novio se da compañía de su amigo, pero dirigido a otras mujeres.

(8) Sólo los hombres hacen filosofía y ciencia.

Esta ambigüedad, equívoco o anfibología se debe a los diversos significados de la palabra "hombre" por un lado se refiere al género de manera que se opone a "mujer". Siendo así las cosas tal oración expresa una proposición falsa. Otro de los significados de "hombre" es el que se refiere a la especie. En este sentido el contenido proposicional es verdadero pues no se sabe de otra especie animal que haga ciencia y filosofía.

\subsection{Ambigüedad pragmática-contextual}

Este es el tipo de ambigüedad que resulta debida a diversas razones entre las que se destacan la información contextual, los supuestos, la información compartida, la información familiar o cercana, las inferencias que se pueden desarrollar, el tipo de acto de habla que se está realizando, el objetivo o la intención que se está expresando, entre otros. Veamos los siguientes casos.

(9) Se rompió la muñeca.

Este es un caso que raya entre lo léxico y lo pragmático. Es pragmático en la medida en que requiere de información contextual compartida, de trasfondo y familiar. Esta construcción formaba 
parte del encabezado de un periódico en el que se hablaba acerca de la famosa caída de Juan Gabriel de un escenario. Así, una lectura radica en que con "muñeca" uno puede referirse a la parte del cuerpo que articula la mano con el brazo y que es susceptible de quebrarse a causa de una caída. Sin embargo, con la palabra "muñeca" también se hace referencia a la figura de una mujer hecha de algún material y que sirve para jugar o de adorno, para dirigirse a una mujer de manera cariñosa o a un hombre afeminado. Juan Gabriel tenía la fama de ser un cantante homosexual. De modo que esa construcción puede ser interpretada como que lo que se rompió por la caída fue Juan Gabriel que era homosexual y al cual se puede identificar con la palabra "muñeca". En definitiva, en esa situación y para el caso de quien se cayó se produce la ambigüedad intencionalmente.

(10) ¿Puede abrir y cerrar la mano?

Este es un tipo de caso muy frecuente, consiste en no distinguir si se trata de una pregunta o una solicitud. De este modo, se puede responder simplemente que sí o que no, pero también se puede ejecutar la acción de abrir y cerrar la mano, dependiendo de si es una pregunta o una solicitud.

(11) En el 2015 mi novia trabajaba en un table dance.

Este caso es pragmático porque se presta para dos tipos de inferencias que conducen a diferentes interpretaciones. Por un lado, se puede inferir que se trata de la misma novia del 2015 que era la que trabajaba en el table dance y tal vez ahora trabaja en otra cosa. Por otro lado, se infiere que se trata de otra novia a la que se tiene en el presente donde se da el acto de emisión, de modo que la novia anterior, pero no la actual es la que trabajaba en el table dance.

\subsection{Ambigüedad lógica}


Este tipo de ambigüedades se dan y/o se resuelven en función de las relaciones lógico-matemáticas presentes para expresar un contenido semántico dado. El siguiente caso es un ejemplo de este tipo de ambigüedades.

(12) Tres amigos trajeron dos botellas.

Para comenzar tiene lugar un proceso pragmático de enriquecimiento para que en la proposición se exprese que las botellas son de algún tipo de bebida alcohólica. Y las relaciones lógico-matemáticas tienen que ver con la distribución de las dos botellas. Por un lado se puede interpretar que entre los tres amigos llevaron dos botellas en total. Por otro lado que cada uno de los tres amigos llevaron dos botellas, lo que en total daría seis botellas.

Las ambigüedades aportan evidencia de que interpretar una construcción lingüística es una tarea que requiere de diversas operaciones lingüísticas e intelectuales o cognitivas. Jakobson (1963, p. 94) mantenía que para el hablante la ambigüedad no existe, ya que en los contextos de emisión se opta por la interpretación más conveniente (a ese contexto lingüístico) debido a que se cuenta con la información relevante para ello, bajo el supuesto de que dicha expresión lingüística emitida sea ambigua de acuerdo a alguno de los niveles de organización lingüística antes vistos. De este modo, la ambigüedad pasa desapercibida. Sin embargo, para el caso de las ambigüedades intencionales se precisa que ésta exista en la cabeza del hablante. Cualquiera que sea el caso, significa que el oyente es quien enfrenta el problema de desambiguar para poder lograr acoplarse a lo que el hablante emitió y así poder lograr un sentido compartido, que es cuando se puede hablar de significado de una construcción lingüística de manera cabal. Por otro lado, la información proporcionada por el contexto en que se emite un texto influye o determina la interpretación que el oyente selecciona entre las diferentes posibilidades. Es decir, el contexto es esencial en la desambiguación. Ahora bien, las diversas interpretaciones que se puedan tener de una sola construcción lingüística son disyuntivas e incompatibles, esto es que una interpretación excluye a la otra. De esto se sigue que el contexto en que ocurre la emisión de una construcción lingüística que 
de manera aislada posee más de un contenido semántico orienta de manera fundamental qué interpretación es la más adecuada. Una vez tomada una interpretación las otras quedan excluidas.

Lo expuesto en este apartado es evidencia para respaldar nuestra primer premisa:

(1) el contexto de una emisión lingüística ambigua determina una de sus interpretaciones, pese a que dicha ambigüedad pueda explicarse en virtud de alguno de los niveles de organización de la lengua.

Como se mostró más atrás, en cualquier lengua natural no hay palabras que posean un solo significado ni significados privativos de un solo ítem léxico, lo mismo sucede con construcciones más complejas, esto da pie a ambigüedades e imprecisiones. En contraste, se suele pensar que el lenguaje científico, debido a su formalidad, rigurosidad y precisión carece al menos idealmente de tales casos. Sin embargo, a continuación, daremos cuenta de un casopeculiar de algo que puede ser concebido como ambigüedad en la mecánica cuántica, cuya clarificación lógica y significación inequívoca exigen, como en el caso anterior, la información del contexto para evitar ineludibles contradicciones.

\section{La interpretación de Copenhague: el comportamiento dual de los fenómenos atómicos}

En el campo de la física, la mecánica cuántica da cuenta del comportamiento de los fenómenos atómicos o microfísicos. Si bien esta teoría cuenta con diversas interpretaciones que son inconsistentes entre sí y algunas de ellas muy polémicas, la denominada interpretación ortodoxa o de Copenhague surgió en el año de 1927 como resultado de las reflexiones de los físicos Werner Heisenberg y Niels Bohr ${ }^{1}$. De acuerdo con esta descripción, los objetos microfísicos como el electrón o el fotón tienen un comportamiento corpuscular u ondulatorio dependiendo del contexto

\footnotetext{
${ }^{1}$ Si bien esta interpretación se conoce como la interpretación canónica de la teoría cuántica, debido a que superó las diversas objeciones que durante varias décadas intentaron refutarla y se estableció como la interpretación dominante particularmente entre las décadas de 1930 a 1950, en la actualidad no necesita ser recuperada de forma íntegra para dar cuenta de los fenómenos atómicos.
} 
experimental que determina el fenómeno que estamos observando. Ambas descripciones son necesarias para dar cuenta de los fenómenos de forma exhaustiva, pero también son mutuamente excluyentes. Por lo tanto, tenemos un tipo de caso de ambigüedad en la que el contexto desempeña un papel fundamental en la desambiguación y selección de la interpretación. Haciendo una analogía con lo expuesto en la sección anterior, el contexto observacional haría la función semejante de cualquiera de los niveles lingüísticos analizados que determinan y sirven para solucionar la ambigüedad lingüística. A continuación, presentamos más claramente los argumentos para justificar esta afirmación, para ello se recurrirá a algunos datos históricos².

La teoría cuántica completó de forma clara y consistente sus estructuras formales, establecidas en la mecánica matricial y la mecánica ondulatoria, hacia el año de 1926. No obstante, la descripción cualitativa de lo que ocurría en los experimentos microscópicos no era en absoluto clara. Durante los meses que siguieron a la elaboración de la teoría los físicos intentaron elegir entre la naturaleza corpuscular u ondulatoria del electrón. Sin embargo, muy pronto se dieron cuenta de que elegir una de las dos categorías, onda o partícula, para describir cualitativamente los procesos del mundo atómico llevaba a contradicciones insalvables ${ }^{3}$.Por tanto, la inevitable conclusión era que

\footnotetext{
${ }^{2}$ Parte de esta explicación se desarrolla también en el artículo "Similitudes entre física cuántica y la psicología de Jung: el caso de la sincronicidad o de cómo fracasa una analogía" con el objetivo de comparar el carácter acasual de la teoría y el concepto de sincronicidad de Jung (Hernández 2016, p. 77-79).

${ }^{3}$ La teoría de Schrödinger partía de la idea de que los electrones eran ondas y no partículas. Por tanto, Schrödinger pensó que los modos de vibración armónica de los electrones descritas por su función de onda y sus transiciones continuas sustituirían los saltos cuánticos entre estados contenidos en los métodos matriciales que describía la mecánica que anteriormente había elaborado Heiseneberg. De esta forma, no sólo evitaba la extraña discontinuidad de los procesos físicos que implicaba el postulado de Planck y el método de Heisenberg, sino que lograba una representación más intuitiva de los fenómenos que la que proporcionaba este abstracto método de matrices (Cassidy 1992, p.212-214).

Sin embargo, esto represento dificultades inevitables. En primer lugar, resultaba poco plausible que un paquete de ondas concentrara una carga eléctrica casi puntual, como lo hace el electrón, pues este grupo de ondulaciones se debe dispersar rápidamente y extenderse en una región amplia del espacio. En segundo lugar, en el momento de la observación, la ecuación de onda repentinamente deja de ser válida, a lo que se le denomina el "colapso de la función", y posteriormente el sistema obedece de nuevo a dicha ecuación, pero con nueva información. Este hecho resultaba inexplicable si trata de una onda física que se propaga en el espacio de forma continua, como afirmaba Schrödinger (Rioja 1995, p. 255). En tercer lugar, la ecuación de onda se define en un espacio abstracto (matemático) de configuración, cuyo número de dimensiones depende del número de grados de libertad del sistema, por lo que no se trata del espacio físico donde se espera que se propague una ondulación material. Adicionalmente, la función de onda
} 
no existía una interpretación del formalismo que fuera consistente entre las ecuaciones de la teoría y las descripciones de los fenómenos haciendo uso de los conceptos clásicos de onda y partícula que se habían utilizado, hasta finales del siglo XIX, sin ningún problema para describir la radiación y la materia, respectivamente. En esta nueva situación, algunos experimentos parecían ser descritos adecuadamente con el modelo corpuscular y otros con el modelo ondulatorio de forma paradójica: si había experimentos en los que parecía posible contar partículas usando un contador Geiger ${ }^{4}$ o fotografiar sus trayectorias en una cámara de Wilson ${ }^{5}$, existían otros fenómenos como la interferencia y la difracción que llevaban de nuevo a la idea de las ondas de materia.

Estas dificultades interpretativas fueron resueltas unos meses más tarde con el surgimiento de la Interpretación de Copenhague. Aunque dicha interpretación no es una descripción exhaustiva ni homogénea de todos los aspectos de los fenómenos atómicos, sí establece los principios de complementariedad y de indeterminación como base explicativa de éstos. De tal forma que, estos

es una función compleja a la que es difícil atribuirle un significado físico, pues normalmente sólo a los números reales les asociamos cantidades físicas (Rioja 2002, p. 138).

En este contexto, el físico Max Born se dio cuenta de que era posible interpretar el cuadrado del módulo de la función de Schrödinger como una probabilidad de densidad de electrones. De tal forma que, lo que indicaba físicamente la función era la probabilidad de encontrar al electrón en un estado específico, pero no proporcionaba su estado preciso (Jammer 1974, p. 40). Como consecuencia, a primera vista parecía plausible que, si los físicos adoptaban la interpretación probabilista de la función de onda, entonces la concepción corpuscular del electrón podía ser recuperada Pese a la plausibilidad de esta hipótesis, otras dificultades aparecieron. Por un lado, las ondas de probabilidad presentaban interferencias de probabilidades como sucede con las ondas físicas. Por otro, la función de onda obedece al principio de superposición y se produce un estado nuevo que se "solapa" con los anteriores. En este caso, hay una superposición de estados en todos los estados y lugares para los que la función de onda atribuye un valor distinto de cero (Rioja 2002,p. 141). Ante esta dificultad la interpretación tampoco podía ser reducida a la noción de partícula y la descripción de los fenómenos microfísicos carecía de una descripción clara y unívoca.

${ }^{4} \mathrm{El}$ contador Geiger es un instrumento que permite medir la radiactividad de un objeto o lugar. Está formado por un tubo con un hilo metálico a lo largo de su centro. El espacio entre ellos está aislado, relleno de un gas y con una diferencia de potencial entre el hilo y el tubo. Un ion o electrón penetra en el tubo, desprende electrones de los átomos de gas y, debido al voltaje positivo del hilo central, éstos son atraídos hacia el hilo. Al hacer esto ganan energía, colisionan con los átomos y liberan más electrones. Así, el proceso se convierte en una "avalancha" que produce un pulso corriente detectable.

${ }^{5}$ La también denominada cámara de niebla, se usa para detectar partículas de radiación ionizante. Se trata de un entorno cerrado que contiene vapor de agua superenfriado y supersaturado. Cuando una partícula cargada de suficiente energía interacciona con el vapor, lo ioniza. Los iones resultantes actúan como núcleos de condensación, alrededor de los cuales se forman gotas de líquido que dan lugar a una niebla. Al paso de las partículas se va produciendo una estela o traza, debido a los numerosos iones producidos a lo largo de su trayectoria. 
dos principios constituyen un nuevo marco lógico y conceptual de las descripciones cuánticas. Para ello toman como punto de partida la recuperación de los conceptos clásicos de la física macroscópica para explicar los fenómenos atómicos, pero estableciendo nuevos márgenes, tanto teóricos como experimentales.

En primer lugar, es justamente Bohr quien se da cuenta de que el dilema entre ondapartícula al que nos hemos brevemente referido es irreducible y que habrá que convivir con él refinando el lenguaje de la física clásica para que diese cabida en la nueva teoría a la coexistencia de ambas concepciones (Selleri 1986, p. 100). Así, tomando como requisitos indispensables la posibilidad de dar cuenta de todos los experimentos concebibles (completud observacional), así como la recuperación de las categorías clásicas de onda y partícula, el físico danés elabora el principio de complementariedad. Su punto de partida consiste en que la esencia de la teoría cuántica es el postulado cuántico de Planck, el cual atribuye un elemento de discontinuidad esencial a todo proceso atómico en contraste con la física clásica. Este elemento de discontinuidad implica que la interacción o el intercambio de energía entre el instrumento de medida y el objeto no puede ser despreciada o reducida arbitrariamente ${ }^{6}$, por tanto, el estado de un sistema no puede ser definido de forma clásica, independientemente de los medios de observación (Jammer 1974, p. 90).

Por un lado, si la observación no puede ser hecha sin despreciar ciertas interacciones con el agente de medida, la noción de causalidad no puede ser aplicada, pues el sistema no se encuentra aislado y la energía total no se mantiene constante. Como consecuencia, no podemos aplicar los teoremas de conservación indispensables para este tipo de descripción.

Por otro lado, la discontinuidad genera que los conceptos espacio-temporales pierdan su sentido ordinario. Todo ello lleva a la conclusión de que la complementariedad tiene como elemento central la imposibilidad de hacer una descripción espacio-temporal simultáneamente con

\footnotetext{
${ }^{6}$ En el contexto clásico la interacción entre el instrumento y el objeto puede ser en principio despreciada gracias a que el principio de continuidad física permite suponer que ésta es en arbitrariamente reducible hasta que se vuelve tan pequeña como queramos o tiende a cero. Por tanto, en este caso podemos decir justificadamente que dicha interacción no afecta la medida realizada sobre el objeto y que éste es independiente de los medios de observación.
} 
una descripción causal de los fenómenos atómicos. En su lugar, debemos concebirlos como aspectos complementarios que se excluyen mutuamente en la descripción de los experimentos.

Es decir, en la descripción cuántica hay una relación entre la renuncia a la causalidad y la imposibilidad de distinguir entre un fenómeno y su observación. La única forma de conservar la causalidad es prescindir de la descripción espacio-temporal para preservar el sistema cerrado. Por el contrario, al observar el sistema lo podemos describir en el espacio y el tiempo, pero debemos desistir de la descripción dinámica conforme al principio de causalidad. El uso de un dispositivo experimental para hacer una de las descripciones excluye la posibilidad de usar otro dispositivo simultáneamente para hacer la correspondiente, como se suele hacer en el caso clásico. De tal forma que se asociará dicha descripción complementaria con las nociones de partícula y onda, afirmando que estas dos categorías y sus respectivas variables aparecerán en el comportamiento de los fenómenos físicos de forma igualmente alternada.

Así, Bohr establece la dualidad entre onda y partícula para interpretar los fenómenos de la teoría cuántica, evitando las contradicciones e inconvenientes de las nociones clásicas. Todos los hechos sobre la luz y la materia deben ser explicados en términos de uno de estos dos conceptos, pero no de los dos simultáneamente, dado que tienen propiedades excluyentes. El uso de uno u otro concepto está determinado por el contexto experimental en el que se define el fenómeno. Es decir, la explicación de éste exige la descripción completa del montaje experimental y de dicho montaje depende el comportamiento corpuscular u ondulatorio, tanto de la materia como de la radiación. Dicho de otro modo, el formalismo se aplica a los instrumentos y los objetos en conjunto.

De forma más específica, toda medida que pretenda describir la localización espaciotemporal o corpuscular del objeto requiere de un montaje experimental de escalas fijas y relojes, lo cual impide un control del intercambio de energía y momento entre objeto y el dispositivo que utilizamos como marco de referencia. Recíprocamente, una determinación de la energía y el momento implica un dispositivo que impide la coordinación espacio-temporal, porque el 
instrumento no está rígidamente sujeto al marco de referencia. En suma, existe una inevitable incompatibilidad experimental de los aparatos que miden ambos conjuntos de variables.

Ahora bien, esta situación se expresa cuantitativamente en el principio de indeterminación de Heisenberg que establece la imposibilidad para fijar variables cinemáticas y dinámicas simultáneamente, con una precisión arbitraria, en un estado físico ${ }^{7}$.

Es decir, no podemos medir al mismo tiempo determinadas variables, denominadas canónicamente conjugadas, como son posición $(x)$ y velocidad o momento $(p)$ o energía (E) y tiempo (t) respectivamente, más que dentro de los límites cuantitativos que el principio establece a través de la constante de Planck (h). De acuerdo con el físico alemán, el significado de las categorías se puede conservar a reserva de que dicha condición limite su uso y tal límite tiene un carácter teórico, porque se deduce desde el formalismo, y experimental, porque es imposible combinar ambos dispositivos.

Como consecuencia, la indeterminación de una de las variables, posición o velocidad, es señal de que el electrón no puede ser concebido inequívocamente como una partícula o como una onda. Por ello, un objeto unas veces comportará como una partícula, puntual en el espacio y el tiempo, y otras como algo extendido, ondulatoriamente. Con esta interpretación, Bohr y Heisenberg pretenden trasladar la consistencia del formalismo matemático cuántico carente de contradicciones internas al plano del lenguaje ordinario, modificando convenientemente su alcance. Lo central es que el dispositivo experimental completo sirve para definir en términos clásicos y sin ambigüedades las condiciones bajo las cuales aparece uno u otro fenómeno. Las siguientes palabras de Bohr expresan este espíritu interpretativo:

\footnotetext{
${ }^{7}$ En su artículo sobre las relaciones de indeterminación Heisenberg explica que: si $q_{1}$ es la precisión con la que conocemos el valor de $q$ (es decir, el error en la medida) y $p_{1}$ la precisión con la que conocemos el valor de $p$, entonces: $\mathrm{p}_{1} \mathrm{q}_{1} \geq \mathrm{h}$, donde $\mathrm{h}$ es la constante de Planck. Esta desigualdad se deduce la relación mecánico-cuántico fundamental y es una consecuencia de la no conmutatividad de las matrices asociadas a estas variables que son justamente energía y tiempo, o posición y velocidad o momento. Por tanto, dicho principio establece un límite inferior para determinar ambas variables simultáneamente; mientras más precisa es la determinación de la posición, por ejemplo, menos será la del momento y viceversa (Heisenberg 1953, p. 64).
} 
Es en esta situación donde la noción de complementariedad puede suministrar un marco suficientemente amplio como para abarcar la explicación de las regularidades fundamentales de la Naturaleza que no puede incluirse en una imagen única. Ciertamente, los resultados obtenidos bajo condiciones experimentales y definidas $-\mathrm{y}$ expresadas por medio del uso adecuado de los conceptos físicos elementales- agotan toda la información que es posible comunicar en un lenguaje corriente a propósito de los objetos cuánticos (Bohr 1970, p. 16).

De acuerdo con todo lo anterior, ha quedado claro que la redefinición de los conceptos clásicos en la teoría cuántica es esencial para una interpretación precisa y consistente de la teoría. De acuerdo con el propio Heisenberg, si los conceptos como posición y velocidad están restringidos por el propio formalismo, la noción de partícula debe ser revisada. Es decir, si una partícula está definida por tener una localización puntual en el espacio y en el tiempo, no podemos aplicar la noción de partícula para todo momento: al medir la velocidad del sistema la localización queda indeterminada, entonces la definición de partícula no puede ser usada en ese contexto. Esto implica que la exclusión mutua de los montajes para medir una u otra variable es necesaria para la aplicación inequívoca de los conceptos.

Así, el contexto experimental será indicativo de cuando podemos usar cada concepto y dicha indicación está expresada mediante las relaciones de incertidumbre. Atenernos a dicho contexto posibilita una aplicación de los conceptos sin contradicciones. En palabras de Heisenberg:

La necesidad de desbordar los confines de los conceptos clásicos se ha originado sobre todo con la ampliación técnica de nuestro ámbito de experiencias. Los conceptos clásicos no se adaptaban ya a la situación en que la naturaleza nos había puesto. Si una vez vemos al electrón describir su trayectoria como partícula en una cámara de Wilson y otra observamos que se refleja como una onda en una retícula de difracción, entonces el lenguaje de la física no basta para hacer comprensibles estas dos observaciones como derivadas de un hecho único. Resulta pues necesario determinar primero con mayor precisión los puntos en los que los conceptos clásicos pierden su aplicabilidad unívoca (Heisenberg 1962, p. 182). 
Dicho de otro modo, la ambigüedad de los conceptos surge de atribuir propiedades físicas a fenómenos que no permiten distinción precisa entre el comportamiento independiente de sus objetos y su interacción con el dispositivo de medición. Es decir, surge de usar en el nivel microfísico las nociones clásicas de onda y partícula en el marco de las suposiciones generales de la física macroscópica, a saber, que las propiedades de los objetos son independientes de su observación. Sin embargo, si evitamos tales supuestos, que se ven impedidos por el cuanto de acción, y limitamos adecuadamente el uso de tales nociones de acuerdo con el contexto experimental, los conceptos tienen un significado inequívoco y perfectamente definido. El precio que hay que pagar es que no podemos hablar de fenómenos independientes en el sentido clásico.

De esta forma, podemos afirmar que existe un criterio operacional en los conceptos, ya que su significado es definido por la manipulación requerida para establecer bajo qué concepto cae el objeto. En la perspectiva de Copenhague, el concepto del objeto no tiene significado al margen de sus manifestaciones fenoménicas, pues las entidades inobservables carecen de significado (Folse 1985, p. 161). Así, un predicado físico sólo se aplica con significación en un contexto que incluye la medida de la propiedad que tal predicado expresa (Murdoch 1989, p. 148). En definitiva, se recuperan los conceptos que Bohr considera explicativos de la teoría, onda y partícula, pero en función de establecer su rango de aplicación de acuerdo con lo que Heisenberg denomina observable.

Por tanto, con lo expuesto en este apartado se refuerza nuestra segunda premisa:

(2) el contexto de experimentación selecciona una de las interpretaciones del electrón, ya sea como partícula o como onda.

\section{Ambigüedad en el lenguaje de la teoría cuántica y el lenguaje natural.}

Con este ejemplo del lenguaje físico se muestra que, al parecer, la ambigüedad no es algo que sólo ocurre en las lenguas naturales. Para el caso de las lenguas naturales hemos visto que para interpretar una construcción lingüística se requiere de diversas operaciones cognitivas, así como del 
contexto para lograr un sentido compartido y bien definido. Para algunos casos en física, específicamente en lo que respecta al electrón, se expuso que la información proporcionada por el contexto experimental en que se emite una afirmación sobre dicho electrón determina la interpretación entre la onda y la partícula. Por tanto, este contexto resulta esencial en la desambiguación, aun cuando se trata, a primera vista, de un lenguaje científico, riguroso y formal. Asimismo, las diversas interpretaciones que podemos tener sobre los enunciados relativos al objeto de estudio son disyuntivas e incompatibles, de lo cual se sigue que la relación entre el electrón y el dispositivo de medida en el que ocurre la emisión de una construcción lingüística establece la interpretación adecuada de dicho objeto (i.e. su identidad). Al margen de tal relación la emisión no tiene un contenido semántico bien definido o incluso es contradictorio con otras afirmaciones a propósito del mismo objeto.

En otras palabras, hay un paralelismo entre los estudios lingüísticos, relativos a la ambigüedad en las lenguas naturales o idiomas, y los estudios en mecánica cuántica, relativos al comportamiento de los electrones. Pero, ¿podemos explicar dicho paralelismo, además de señalarlo? ¿nos dice algo sobre la ciencia y el lenguaje? ¿cómo interpretarlo? Veámoslo más detenidamente.

Las lenguas naturales o idiomas son una variación del lenguaje que consiste en la facultad de poseer un sistema lingüístico estructurado, esto es, una lengua. Por ello, las lenguas son variaciones del lenguaje. Asimismo, toda lengua tiende a especializarse de acuerdo a la gran cantidad de situaciones de nuestras prácticas cotidianas, en particular, a los propósitos para los que sirven. La lengua se adapta a estas situaciones y gracias a ello se especializa, de ahí resultan lo que se conoce como lenguajes especializados tales como el lenguaje filosófico, matemático, el lenguaje de la física que es el que nos concierne por ahora, entre otros.

Ahora bien, al estudiar las lenguas naturales se encuentra el fenómeno de la ambigüedad y al estudiar al electrón se encuentra el fenómeno de la dualidad o de la explicación dual ondapartícula. Pero, aunque en ambos casos se trate de ambigüedad ésta no viene determinada ni se explica de la misma manera, si bien podemos encontrar cierta relación entre ellas. Lo que 
determina y explica la ambigüedad en la lengua son los diversos niveles de organización de ésta. En el caso de la mecánica cuántica la cuestión puede ser un tanto más complicada y polémica. Como hemos visto, en un primer momento, los físicos encontraron dos estructuras matemáticas que hacen las predicciones correctas sobre las variables de los sistemas atómicos. De tal forma queel lenguaje formal de la teoría es consistente y correcto. Por tanto, el problema de la ambigüedad surge cuando se quiere hacer una descripción cualitativa de dichos resultados a nivel microscópico, haciendo uso de los conceptos clásicos de onda y partícula que surgen en el marco de una teoría con principios de realidad distintos a nivel macroscópico (la continuidad), como hemos visto en el apartado anterior.

En ese escenario, como A. Rioja (1995) apunta, históricamente, se han discutido tres distintas opciones: a) La de restringirse al uso del lenguaje matemático, sin proporcionar una descripción cualitativa de los fenómenos, b) elaborar nuevos conceptos que permitan una descripción sin ambigüedades, y c) redefinir los conceptos clásicos, con las restricciones que determina el formalismo cuántico, tal como lo hicieron Heisenberg y Bohr en la interpretación de Copenhague.

La opción de restringirse al lenguaje formal (a) fue inicialmente defendida por Heisenberg, pero eventualmente abandonada ante la insistencia de Bohr de que la física debe poder describir cualitativamente lo que pasa en el mundo atómico. Aún así, en ocasiones es pragmáticamente utilizada por las comunidades de físicos, para no entrar en cuestiones interpretativas polémicas. La idea de elaborar un nuevo vocabulario (b) ha tenido cierto impulso y desarrollo (Bloch, Feyerabend), sin embargo, estos intentos han resultado infructuosos, ya sea por argumentos que defienden la necesidad o inevitabilidad de que las descripciones físicas estén enraizadas en el lenguaje natural y éste a su vez exige que los objetos estén descritos de forma causal en el espacio y en el tiempo (i.e. a través de las categorías de onda y partícula), como por argumentos que afirman que nuestras formas de percepción tienen un carácter trascendental y requieren de las categorías clásicas. Así, parece poco plausible (y útil) que podamos elaborar conceptos nuevos que no remitan 
de alguna forma a nuestras formas habituales de experiencia, por tanto, a conceptos y significados previos. $^{8}$

Así, la vía de redefinición de los conceptos clásicos (c), tomada por Copenahgue, tiene como premisa la necesidad del lenguaje clásico, tanto porque remite nuestras formas ordinarias de experiencia y con ello al lenguaje natural (refinado), como porque, al menos de acuerdo con Bohr, estas son formas necesarias de nuestra experiencia física. Llamaremos a estos dos tipos de argumentos de orden social y/o pragmático, y de orden trascendental.

De tal forma que, lo que explica y determina la ambigüedad del electrón es, de acuerdo con Heisenberg y Bohr, que debido a los principios de realidad (discontinuidad) en el dominio cuántico, los conceptos clásicos pierden su aplicabilidad unívoca, pero como dichos conceptos son necesarios para la descripción, es el contexto experimental el que la resuelve a través de su comportamiento dual. En suma, la discontinuidad y la inevitabilidad del lenguaje clásico son responsables de esta ambigüedad cuántica.

Lo que queremos resaltar es que en ambos casos se presenta la importancia del elemento contextual para desambiguar y así obtener significados inequívocos. Además, resulta notable de este análisis es que se muestra que la ambigüedad de las expresiones lingüísticas que configuran a la mecánica cuántica sigue siendo un elemento inherente a éstas si importar la rigurosidad, formalidad y precisión con la que se construye dicha teoría. Al menos si seguimos la argumentación de Bohr, dado que las otras dos vías (a) y (b) no han resultado exitosas, pues ni parece que podamos evitar hablar cualitativamente de los fenómenos físicos ni parece viable un esquema conceptual completamente nuevo.

A su vez el carácter dual del electrón nos plantea un problema en relación al lenguaje al que intentaremos dar algo de luz a partir de lo expuesto. Es decir, la interpretación dual onda-partícula del electrón se debe (i) a las teorías que han sido configuradas (por los físicos), así como a los contextos experimentales diseñados desde dichas teorías, (ii) es algo que se hereda de las lenguas

\footnotetext{
${ }^{8}$ A. Rioja (1995) examina críticamente algunos intentos por introducir neologismos en la teoría por parte de autores como Lévy-Leblond (pp. 150-3).
} 
naturales desde las que se construyen las teorías mediante los lenguajes especializados; o (iii) tal vez, así sea la naturaleza del electrón.

Así, una primera aproximación filosófica a este problema podría llevarnos a teorizar sobre la naturaleza de tales ambigüedades. Respecto de la opción (i), los mismos físicos rechazaron tal opción al considerar que la ciencia es un refinamiento del lenguaje natural, por lo que una ruptura radical entre éste y los esquemas conceptuales de las teorías físicas resultaría insostenible. Por lo tanto, sería más plausible que la ambigüedad tuviera origen en el lenguaje natural, heredado al lenguaje de la física. De hecho, como ya vimos, los físicos intentan trasladar la consistencia del lenguaje formal al ordinario modificando su alcance, y en ese salto o transición aparece la ambigüedad. Por tanto, parece haber una conexión en la determinación de ambas ambigüedades, en tal caso se explicaría la aparición de estas en los dos dominios debido a que aun en una teoría tan formal y precisa como intenta ser la física cuántica se precisa el lenguaje natural u ordinario.

Ahora bien, en tal caso, aún permanece la disyuntiva de si se trata de un elemento que se debe a nuestras formas de conceptualizar y entender la naturaleza o si se trata de la estructura misma de ésta que exige ser representada de manera complementaria o dual.

Sobre la primera aproximación, ya dijimos, los mismos físicos y filósofos de la ciencia han debatido en su momento sobre la posibilidad de generar un nuevo lenguaje especializado con nuevas categorías cuya univocidad no exija hacer referencia al dispositivo experimental ${ }^{9}$. En cualquier caso, este enfoque atribuye la ambigüedad exclusivamente a nuestras características o limitaciones cognitivas o lingüísticas, y puede poner en entredicho, la objetividad de la ciencia, al no poder dar cuenta de los fenómenos de forma independiente de los instrumentos de medida ${ }^{10}$.

Sobre la segunda aproximación, si es la propia naturaleza la que presenta de forma inherente un comportamiento dual que simplemente es representada en la teoría cuántica, tal

\footnotetext{
${ }^{9} \mathrm{Al}$ respecto también se puede consultar la discusión que elabora el propio Heisenberg, fuertemente influido por los argumentos de Bohr, en "Cuestiones de principio en física moderna" en Heisenberg, W. Los nuevos fundamentos de la Ciencia (1962) o en "Lenguaje y realidad en la física moderna" en Más allá de la física (1971).

${ }^{10}$ Una de las muchas críticas que se han hecho a esta interpretación afirma que la perspectiva dual de los fenómenos atómicos resulta subjetiva, ya que la descripción corpuscular u ondulatoria depende del dispositivo experimental usado, por tanto, no describe los fenómenos independientemente de sus medios de observación.
} 
perspectiva exige una interpretación representacionista y realista de la ciencia que ha mostrado sus propias dificultades en la filosofía de la ciencia contemporánea ${ }^{11}$. Así, atribuirle este carácter dual a la naturaleza en sí misma sugiere el compromiso con una perspectiva metafísica de la ciencia que presenta fuertes objeciones historicistas, ya que dificulta explicar el cambio científico ${ }^{12}$.

Intentando evitar la disyuntiva anterior, que nos parece infructuosa, sugerimos una interpretación que hace énfasis en el carácter social y pragmático de los lenguajes especializados, aludiendo al primer tipo de argumento de exponen Bohr y Heisenberg, y, al mismo tiempo, evita la dicotomía entre lenguaje y naturaleza. Es decir, si pensamos en los lenguajes especializados como una práctica cultural necesariamente enraizada en el resto de nuestras experiencias, en este caso científicas, se vuelve plausible defender la independencia causal de los fenómenos naturales, al tiempo que reconocemos que nuestras descripciones y justificaciones de éstos dependen de las relaciones inferenciales que se establecen en nuestras teorías. Así, evitamos atribuir la dualidad a la naturaleza o a un rasgo de nuestras estructuras cognitivas o lingüísticas y, al mismo tiempo, se muestra de forma natural el componente pragmático en la estructura de las lenguas. Como dijimos, la naturaleza tiene relaciones causales que son completamente independientes de nuestras formas de descripción, sin embargo, los conceptos y relaciones teóricas con los que damos cuenta de ellas están constituidos por un lenguaje especializado, en tanto práctica cultural, que a su vez se encuentra enraizado y requiere de una forma u otra el lenguaje natural. En este sentido, nuestras teorías dependen de la estructuración de dicho lenguaje, y este se encuentra determinado de manera simultánea por los fenómenos naturales y prácticas sociales.

Ello pone de manifiesto la necesidad de redefinir una noción de objetividad distinta de la clásica, que no se refiere a una descripción independiente del mundo, lo que se torna imposible una vez que abandonamos la dicotomía entre éste y nuestra práctica lingüística. Es decir, que nuestras prácticas lingüísticas están articuladas al mundo, en el sentido en que se acomodan a éste, como el

\footnotetext{
${ }^{11} \mathrm{Al}$ respecto se puede consultar el artículo de Arthur Fine "The Natural Ontological Attitude" en TheShaky Game.

${ }^{12}$ Como es bien sabido, atribuirle estas propiedades de nuestras teorías científicas a la naturaleza en sí misma dificulta dar cuenta de los cambios conceptuales éstas a lo largo de la historia de la ciencia, como han mostrado Thomas Kuhn y Paul Feyerabend, entre otros.
} 
mundo está articulado a nuestras prácticas lingüísticas, en el sentido en que éstas lo asimilan ${ }^{13}$.Una noción de objetividad derivada de las reflexiones aquí expuestas, sobre el lenguaje especializado, los significados y su dependencia al contexto, se refiere más bien a una posibilidad de comunicación y consenso que dé cuenta de los objetos de forma clara, consistente y precisa. En definitiva, se trata de una objetividad que construye un sentido compartido de las oraciones descriptivas de los procesos que, en este caso, permite predecir y controlar los fenómenos de forma exitosa. Algo a lo que el propio Bohr hizo referencia en sus escritos ${ }^{14}$, pero también los filósofos de la ciencia contemporáneos más interesantes.

Con esto, evitamos, tal vez, la disyuntiva aunque no resolvemos de manera definitiva el problema, éste, al menos, queda planteado en esos términos.

\section{Conclusión}

Con lo presentado a lo largo de todo este trabajo sostenemos lo siguiente:

(1) El contexto de una emisión lingüística ambigua determina una de sus interpretaciones, pese a que dicha ambigüedad pueda explicarse en virtud de alguno de los niveles de organización de la lengua.

(2) El contexto de experimentación determina una de las interpretaciones del electrón, ya sea como partícula o como onda, pese a que dicha ambigüedad surge de la necesidad de expresar cualitativamente, mediante el lenguaje clásico enraizado en el lenguaje natural, los resultados del formalismo cuántico, que obedece a diferentes principios de realidad.

\footnotetext{
${ }^{13}$ Esto hace referencia las nociones de asimilación y acomodación en epistemología genética. La idea es que las lenguas surgen en nuestras prácticas cotidianas y en nuestra interacción con el mundo, pero a su vez estas lenguas se ajustan o se adecuan al mundo, lo que promueve el cambio y la diversidad lingüística. Un ejemplo para ilustrar esto: una lengua construye la palabra "árbol" para hablar de los árboles, luego se producen distinciones, esto es la lengua se tiene que acomodar al mundo y se producen "árbol de cerezo", "árbol de mamey", árbol de abedul", etc. Pero, hay ciertas prácticas de nuestras vidas que la lengua las asimila o las comprende en términos de "árbol de la vida", "árbol genealógico", "árbol sintáctico", etc. Y la idea es que hay cierta circularidad o codependencia entre nuestras prácticas en el mundo y la lengua o bien entre la lengua y nuestras actividades en el mundo.

${ }^{14}$ Bohr hacer referencia a una objetividad, justamente, entendida como la posibilidad de comunicar los fenómenos de forma no ambigua.
} 
(3) Considerando lo que se entiende por paralelismo, se sigue que

(4) Hay un paralelismo en la lingüística y en la mecánica cuántica en tanto que es el contexto ya sea de emisión o de experimentación el que, en última instancia, determina una interpretación entre dos o más posibles.

Además, este paralelismo se encuentra conectado, pues la ambigüedad en la mecánica cuántica parece explicarse a partir de la necesidad, social y práctica, de usar siempre el lenguaje natural para describir los fenómenosfísicos. De esta manera, el paralelismo se explicaría como un rasgo heredado del lenguaje natural al lenguaje de la física, ya que es el lenguaje al que, en una primera instancia, recurren todas las disciplinas científicas.

Finalmente, hemos sugerido que la interpretación de esta ambigüedad que nos parece más conveniente es la que evita la dicotomía entre naturaleza y lenguaje, y que concibe a este como una práctica social que incluye a la ciencia, aunque ello implique, como ha ocurrido en la mecánica cuántica el replanteamiento de algunas categorías epistémicas, como el de la objetividad.

\section{Referencias}

Alonso, M. (2011) Sobre los usos figurados: ¿extensiones de una única definición? En Escandell Vidal, M. V., Leonetti, M. \& Sánchez López, C. (eds.) 60 problemas de gramática. Madrid, España: Akal, pp. 340-346.

Bohr, N. (1970): Nuevos ensayos sobre física atómica y conocimiento humano (1958-1962). Madrid: Aguilar.

Cassidy, D.C. (1992): Uncertainty. The Life and Science of Werner Heisenberg. Freeman: New York. Folse, H. (1985): The Philosophy of Niels Bohr: The Framework of Complementarity. Amsterdam: North-Holland.

Heisenberg, W. (1953): “The Physical Content of Quantum Kinematics and Mechanics" en Quantum Theory and Measurement, J.A. Wheeler, W.H. Zurek (Eds.). New Jersey: Princeton University Press. 
Heisenberg, W. (1962): Los nuevos fundamentos de la ciencia. Madrid: Norte y sur.

Heisenberg, W. (1971): Más allá de la física. Madrid: B.A.C.

Hernández, N. (2016) "Similitudes entre física cuántica y la psicología de Jung: el caso de la sincronicidad o de cómo fracasa una analogía." En Sincronía. Revista electrónica de Filosofía, Letras y Humanidades. XX(70 Junio-Diciembre), pp, 71-102.

Jakobson, R. (1963): Essais de Linguistique générale I. Paris: Minuit. Jammer, M. (1974): The Philosophy of Quantum Mechanics: The Interpretation of Quantum Mechanics in Historical Perspective. New York: John Wiley and Sons.

Löbner, S. (2002): Understanding Semantics. London \& New York: Routledge.

Murdoch, D. (1989): Niels Bohr's Philosophy of Physics, Cambridge Universitiy Press: Cambridge.

Rioja, A. (1995): “La dualidad onda-corpúsculo en la filosofía de Max Born”. Themata: Revista de filosofía. (14), pp.251-284.

Rioja, A. (2002): "Sobre ondas y corpúsculos: un punto de vista lingüístico" en Física cuántica y realidad, Rivadulla A., Mataix C. (Eds). Universidad Complutense de Madrid: Madrid, pp. 135154.

Selleri, F. (1983): El debate de la teoría cuántica. Madrid: Alianza. 